\title{
EL USO DEL ANÁLISIS DE INSUMO-PRODDUCTO EN UN MODELO ECONOMÉTRICO DE LA ECONOMÍA MEXICANA
}

\author{
ROGELIO MONTEMAYOR S. \\ $\mathrm{y}$ \\ JESÚS A. RAMÍREZ
}

\section{INTRODUCCIÓN}

EL ESTUDIo presente trata sobre la vinculación de una matriz de insumoproducto con un modelo econométrico de la economía mexicana. El análisis de insumo-producto agrega una nueva dimensión a los modelos de sistemas económicos al considerar las interrelaciones y flujo que ocurren entre sectores de la economía. En algún grado estos se diluyen en el sistema de cuentas nacionales que sirve de base para la construcción del modelo. Al seguir las ideas originales desarrolladas en el modelo econométrico de la Brookings ${ }^{1}$ y el trabajo de R. Preston, ${ }^{2}$ se establece la conexión entre el cuadro de insumo-producto de 1960 con una versión revisada del modelo de predicción DIEMEX-WFFA de la economía mexicana. $^{3}$ El modelo de insumo-producto se integra en forma global con el macro-modelo para resolver un modelo a partir del otro y viceversa. Una vez logrado esto se utiliza el modelo completo para simular medidas de política. Sin embargo, en este trabajo se da atención especial a las políticas que implican cambios en la tecnología como se representan en el modelo de insumo-producto.

${ }^{1}$ F.M. Fisher, L.R. Klein y Y. Shinkai, "Price and Output Aggregation in the Brookings Econometric Model," The Brookings Quarterly Econometric Model of the United States, J.S. Duesenberry et al. (Comps.), Chicago, Rand McNally \& Co., 1965.

2 R.S. Preston, "The Wharton Annual and Industry Forecasting Model," Philadelphia, Economic Research Unit, Wharton School, Universidad de Filadelphia, 1972, Studies in Quantitative Economics, Núm. 7, pp. 14-20.

"Véase A. Beltran del Río, "A Macroeconomic Forecasting Model for Mexico: Specification and Simulations", tesis doctoral inédita, Universidad de Filadelfia, 1973; véase ambién R. Montemayor, "An Econometric Model of the Financial Sector: The Case of Mexico", tesis doctoral inédita, Universidad de Filadelfia, 1974. 
II. METOdología $Y$ PROBLEMAS DE VINCULACIÓN DEL ANÁlisis DE INSUMO-PRODUCTO CON EL MODELO MACROECONOMÉTRICO

Un esquema en el que se muestran las interrelaciones que existen entre "registros" interindustriales y "cuentas" del ingreso nacional, tanto por el lado de los gastos como de los ingresos, aparece en la gráfica 1.

Antes de entrar en detalles sobre la manera de cómo hacer la conexión, es conveniente hacer explícitas las identidades involucradas en la gráfica.

En sentido horizontal se pueden estabelcer la siguiente igualdad:

$$
\sum_{i=1}^{n} X_{i j}+\sum_{k=1}^{n} F_{i k}=X_{i} \quad(i=1, ., n)
$$

Que expresa que la suma de las ventas de la industria $i$ a cada una de las restantes (inclusive a ella misma, las $X_{i j}$ ) más la suma de las ventas a cada una de las categorías de la demanda final $\left(F_{i k}\right)$ : consumo, formación de capital, etc., es equivalente a la producción bruta de la industria $i$.

En sentido vertical, tenemos la siguiente igualdad:

$$
\sum_{i=1}^{n} X_{i j}+\sum_{k=1}^{w} Y_{k j}=X_{j} \quad(j=1, \ldots, n)
$$

O sea, que la suma de las compras que efectúa la industria $j$ a todas las demás, más la suma de los pagos a los $w$ factores de la producción equivale a los insumos totales utilizados por la industria $j$.

También puede verse que los totales de renglón son iguales a los totales de columna:

$$
\sum_{i=1}^{n} X_{i}=\sum_{j=1}^{n} X_{j}=\text { Producción bruta }
$$

Si se consideran las entregas hechas por cada industria a cada uno de los sectores de demanda final, se tiene:

$$
\sum_{i=1}^{n} F_{i j}=G_{j}=\text { demanda final total } j,(j=1, \ldots, n)
$$

y si se consideran los pagos a los factores de la producción:

$$
\sum_{j=1}^{n} Y_{i j}=Y_{i}=\text { pago total al factor } i(i=1, \ldots w)
$$

También se conoce que los gastos totales son iguales al ingreso total:

$$
\sum_{j=1}^{m} G_{j}=\sum_{i=1}^{w} Y_{i}=P I B
$$


Gráfica 1

RELACIONES ENTRE TRANSACCIONES INTERINDUSTRIALES, DEMANDA FINAL Y PAGOS A LOS FACTORES

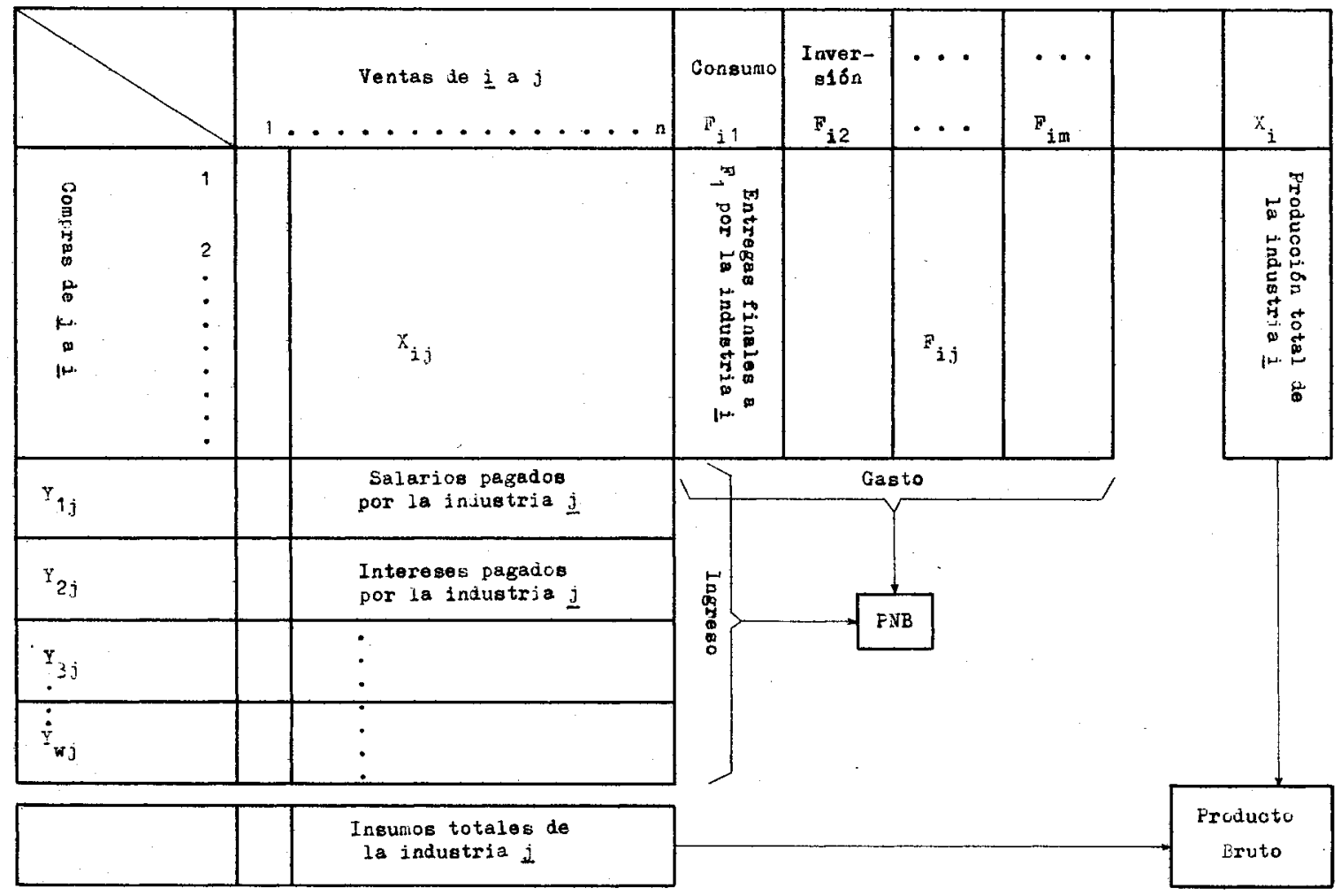


Para cada industria se tienen las siguientes identidades:

$$
\begin{aligned}
\sum_{k=1}^{m} F_{i k}=F_{i}= & \text { Entregas totales de la industria } i \\
& \text { a demanda final }(i=1, \ldots, n) \\
\sum_{i=1}^{w} Y_{i j}=Y_{j}= & \text { Pago total a los factores de la producción } \\
& \text { o valor agregado por la industria } j(j=1, \ldots, n)
\end{aligned}
$$

Si se sustituye (7) en (1) para cada industria o sector, se tiene:

$$
\sum_{j=1}^{n} X_{i j}+F_{i}=X_{i} \quad(i=1, \ldots, n)
$$

Si se supone que:

$$
\frac{X_{i j}}{X_{j}}=a_{i j}
$$

Esto significa que el producto de la industria $j$ es proporcional a los insumos que utiliza de la $i$. Entonces (9) se puede expresar como:

$$
\sum_{j=1}^{n} a_{i j} X_{j}+F_{i}=X_{i} \quad(i=1, \ldots, n)
$$

$\mathrm{O}$ en forma matricial:

$$
\begin{array}{ccccc}
a_{11} \ldots a_{1 j} \ldots a_{1 n} & X_{i} & F_{i} & X_{i} \\
\cdot & \cdot & \cdot & \cdot & \cdot \\
\cdot & \cdot & \cdot & \cdot & \cdot \\
\cdot & \cdot & \cdot & \cdot \\
a_{n 1} \ldots a_{n j} \ldots a_{n n} & X_{j} & F_{j} & X_{j} \\
& & \cdot & \cdot & \cdot \\
& & X_{n} & \dot{F}_{n} & \dot{X}_{n}
\end{array}
$$

y en forma simplificada:

$$
A X+F=X \text { o bien } X=(1-A)^{-1} F
$$

Si $F$ es conocida, se puede predecir de qué magnitud debe ser el sector de la producción bruta para satisfacer una demanda final dada expresada en el sector $F$.

Uno de los primeros aspectos que es necesario afrontar al tratar de vincular la demanda y la producción mediante un modelo interindustrial, es el nivel de agregación de la demanda y de la producción, ${ }^{4}$ así como disponer de una transmisión adecuada de un lado al otro. En nuestro caso se tiene una desagregación de 45 sectores para la producción pero

${ }^{4}$ Véase R. Preston, loc cit., p 14. 
sólo seis categorías de demanda final; entonces hemos reducido el cuadro a 15 sectores para utilizarlo con los seis en que se desagrega la demanda final.

Los 15 sectores en que se desagrega la economía son:

1. Agricultura, ganadería, pesca y explotación forestal;

2. Minería;

3. Extracción y refinación de petróleo;

4. Alimentos, bebidas y tabaco;

5. Textiles y vestuario;

6. Productos de madera, muebles e industria editorial;

7. Química, hule y plásticos;

8. Fabricación de productos minerales no ferrosos;

9. Industrias metálicas básicas;

10. Fabricación y reparación de productos metálicos;

11. Construcción;

12. Electricidad;

13. Comercio;

14. Transporte;

15. Servicios;

$\mathrm{Y}$ los seis rubros de demanda final:

1). Consumo privado;

2). Consumo público;

3). Consumo de los turistas;

4). Exportaciones;

5). Inversión fija total;

6). Cambios de inventarios.

Veamos ahora los detalles de la vinculación de la contabilidad de insumo-producto con el modelo macroeconómico.

Existen tres clases de problemas que se presentan cuando se intenta pasar de las cuentas de ingreso nacional (que son la base de los modelos macroeconómicos) a la contabilidad de las transacciones interindustriales. En primer lugar, no se dispone de series de tiempo sobre las entregas de cada industria a la demanda final, lo que se tiene son los componentes de demanda final del producto interno bruto $(P I B)$, o sea consumo, formación de capital, etc.

Sin embargo, esta información se puede obtener para el año en el que se dispone de una matriz de requerimientos directos. Al establecer los supuestos de proporcionalidad y constancia hechos antes, se pueden transformar los componentes del PIB en entregas a la demanda final por industria:

$$
\frac{F_{i j}}{G_{j}}=h_{i j} \quad(i=1, \ldots n ; j=1, \ldots m)
$$


O sea que el monto de producción que la industria $i$ vende a la categoría $j$ de demanda final $F_{i j}$ es una proporción constante $\left(h_{i j}\right)$ de la demanda final $\left(G_{j}\right)$.

$y$ en forma compacta:

$$
F=H G
$$

donde:

$$
\begin{aligned}
F= & \text { vector }(n \times 1) \text { de entregas a demanda final por industria; } \\
H= & \text { matriz }(n \times m) \text { de coeficientes de distribución industrial o sec- } \\
& \text { torial por categorías de demanda final (se obtiene para el año } \\
& \text { a que corresponde el cuadro de insumo-producto); }
\end{aligned}
$$

$G=$ vector $(m \times 1)$ de demanda final por categoría de gasto.

El segundo problema consiste en lo siguiente: en contabilidad nacional se trabaja con conceptos de valor agregado $(Y)$ mientras que en el análisis de insumo-producto se manejan conceptos de producción bruta. La diferencia entre los dos son las transacciones intermedias.

La relación entre los dos conceptos se puede establecer como sigue: Valor agregado $=$ producción bruta menos compras de bienes intermedios. En forma algebraica:

$$
Y_{i}=X_{j}-a_{1 j} X_{j}-a_{2 j} X_{j}-, \ldots,-a_{n j} X_{j}
$$

o bien:

$$
Y_{j}=\left(1-\sum_{i=1}^{n} a_{i j}\right) X_{j} \quad(j=1, \ldots n)
$$

y sea

$$
1-\sum_{i=1}^{n} a_{i j}=b_{j j}
$$

Entonces (16) puede expresarse en forma matricial:

$$
Y=B X
$$

Donde $B$ es una matriz diagonal con los elementos $b_{j j}$ ya definidos y donde los elementos restantes son ceros.

$\mathrm{Al}$ sustituir (12) y (14) en (17) se tiene:

$$
Y=B(I-A)^{-1} H G
$$

Sea $B(I-A)^{-1} H=C$. O sea una matriz con tantos renglones como sectores en la matriz $(A)$ de requerimientos directos y con tantas columnas como categorías componentes de destino del PIB. 
En nuestro caso se tienen 15 sectores y seis categorías de consumo final. Al despejar $Y$ en (18) se tiene:

$$
\begin{aligned}
& Y_{1}=c_{11} G_{1}+c_{12} G_{2}+\ldots+c_{1 m} G_{m} \\
& \text { - } \cdot \text {. }
\end{aligned}
$$

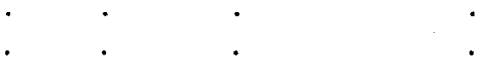

$$
\begin{aligned}
& \text {. . . } \\
& Y_{n}=c_{n 1} G_{1}+c_{n 2} G_{2}+\ldots+c_{n m} G_{m}
\end{aligned}
$$

Sin embargo, la matriz $C$, dadas las propiedades de las matrices $\boldsymbol{A}$ y $\boldsymbol{H}$, tiene la propiedad: $5 \sum_{i=1}^{n} c_{i j}=1$

Esta propiedad implica que:

$$
\sum_{i=1}^{n} Y_{i}=\sum_{i=1}^{m} G_{i}=P I B
$$

Así, dada la matriz $(A)$ de coeficientes técnicos y la matriz $H$ de distribución industrial de las demandas finales, podemos establecer el vínculo entre las diversas categorías de demanda final $\left(G_{i}\right)$ y el producto que se genera o valor agregado por sector $\left(Y_{j}\right)$, vínculo en el que se toma en cuenta la estructura interindustrial de la economía.

Resta sin embargo un tercer problema: se ha establecido implícitamente como supuesto que las matrices $A$ y $H$ son constantes en el tiempo, aunque esto no es así en la vida real. La tecnología y los gustos cambian. Sólo es razonable esperar que $a_{i j}$ y $h_{i}$, cambien en el tiempo, aunque tales cambios puedan ser pequeños y graduales. Esto junto con el hecho de que en México tales matrices, las que existen, se han construido solo cada 10 años, originan que nuestras proyecciones hechas a partir de las matrices dadas $A$ y $H$, contengan un elemento de error.

Existen diversas maneras en las cuales se puede manejar el problema. Una podría ser tratar cada uno de los elementos de la matriz $G$ en el modelo. Sin embargo, esto no es práctico dada la disponibilidad actual de datos específicos referentes a las transacciones interindustriales.

Un segundo camino, más práctico, consiste en utilizar las series de tiempo del vector $G$ y dada la matriz $C$ mediante $(18)$, con lo que se puede estimar una serie de vectores $Y$ :

$$
\widehat{Y}_{t+i}=C G_{t+i} \quad(i=1, \ldots, T)
$$

Estos $\widehat{Y}_{t+i}$ pueden compararse con los vectores reales $Y$ del mismo período y obtenerse una serie de vectores residuales:

$$
Y_{t+i}-\widehat{Y}_{t+i}=U_{t+i} \quad(i=1, \ldots, T)
$$

${ }^{5}$ Ibid, pp. 16-18. 
Los factores que originan los cambios en $C$ son los mismos que dan lugar a los errores observados $U_{t+i}$; se puede así probar esos errores en el modelo. Hay $n$ ecuaciones por estimar, número considerablemente menor que el de las ecuaciones que tendríamos de haber tratado cada uno de los elementos de la matriz $C$ en el modelo. En este caso, nuestras proyecciones serán hechas de acuerdo con la fórmula siguiente:

$$
\hat{Y}_{F}=Y_{F}+U_{F}
$$

En donde $Y_{F}$ se basa en las proyecciones del vector $G$ que proviene del macromodelo de acuerdo con $(21) ; U_{F}$ serán las proyecciones de los errores, basadas en el modelo que se desarrolla para los errores observados en el pasado.

Sin embargo, aunque queda por determinar el vector $U$, existen varios modos de hacerlo. Una manera sería seguir el enfoque de Fisher, Klein y Shinkai, ${ }^{6}$ que utilizan modelos autorregresivos. R. Preston ${ }^{7}$ utiliza dos modelos de tal tipo:

1. $U_{i t}=f\left(U_{i t-1}, t\right)+e_{i t} \quad(i=1, \ldots, n)$

2. $U_{i t}=f\left(U_{i t-1}, U_{i t-2}, t\right)+e_{i t} \quad(i=1, \ldots, n)$

Sin embargo, si se quiere mantener la identidad (20), debe utilizarse el mismo coeficiente de regresión para cada error de las ecuaciones. De otro modo tendría que utilizarse un método para asignar las discrepancias finales.

En el caso dẹ México, se utilizó un modelo ligeramente diferente y después se obtuvieron los errores observados para el período 1951-1971. Fueron obtenidas las componentes principales para los errores de los 15 sectores. Se observó que las primeras tres componentes principales explican el $94.1 \%$ de la varianza total de la serie original; al agregar la cuarta se observa sólo un pequeño incremento $(2.1 \%)$. El modelo utilizado fue el siguiente:

$$
U_{i t}=f\left(P C 1_{t-1}, P C 2_{t-1}, P C 3_{t-1}\right)+e_{i t} \quad(i=1, \ldots, n)
$$

en donde:

$$
\begin{aligned}
& P C 1_{t-1}=\text { primera componente principal con retardo de un período } \\
& P C 2_{t-1}=\text { segunda componente principal con retardo de un período } \\
& P C 3_{t-1}=\text { tercera componente principal con retardo de un período. }
\end{aligned}
$$

Los méritos de este enfoque son dobles: por una parte, se mantiene la identidad del valor agregado y de la demanda final totales (20), con el mismo regresor (las primeras tres componentes) utilizado para cada una de las 15 ecuaciones de error; por otra, con el supuesto de que las

- F.M. Fisher, L.R. Klein y Y. Shinkai, op. cit.

${ }^{\top}$ R.S. Preston, op. cit., pp. 19-20. 
componentes principales son combinaciones lineales de errores, el uso del retraso de un período facilita en gran medida la extrapolación de las componentes principales y los errores en el futuro.

Así, las ecuaciones del valor agregado utilizadas en el modelo serán de la forma:

$Y_{i t}=C_{i 1} G_{1 t}+C_{i 2} G_{2 t} 1+C_{i 3} G_{3 t}+C_{i 4} G_{4 t}+C_{i 5} G_{5 t}+C_{i 6} G_{6 t}$

$+L_{0 i}+L_{1 i}+P C 1_{t-1}+L_{2 i} P C 2_{t-1}+L_{3 i} P C 3_{t-1}(i=1, \ldots, 15)$

En donde:

$Y_{i t}=$ Valor agregado en el sector $i$

$C_{i j}=$ Elemento $i j$ de la matriz $\mathrm{C}$

$G_{i t}=$ Elemento $i$ de la categoría de demanda final en el tiempo $t$

$L_{k i}=$ Coeficiente de regresión de (24)

Las ecuaciones en (25) reemplazan las ecuaciones del valor agregado en el macromodelo. Sin embargo, el modelo contiene sólo tres sectores: agrícola o primario; industrial; y terciario. Por lo tanto, las 15 ecuaciones de valor agregado deben agregarse en tres.

El macromodelo tiene también una sección de demanda final. ${ }^{8}$ Aquí existe una ecuación para cada categoría de demanda final. La mayoría de ellas relacionadas directa o indirectamente con la producción- la inversión privada es una ecuación modificada con acelerador flexible. Así completamos el círculo: la producción es explicada mediante la matriz $C$ en términos de demanda; el valor agregado retroalimenta los requerimientos de fuerza de trabajo, salarios y precios y la demanda misma. Se requiere el macromodelo (los valores de $G$ ) para resolver el modelo insumo-producto $(\operatorname{los} \mathrm{Y})$ y viceversa. En el algorítmo de solución se obtienen en forma simultánea los valores de $Y$ y $G$.

En el cuadro 1 se proporcionan los resultados obtenidos para los errores o ecuaciones residuales.

\section{Simulación de políticas}

A menudo, las implicaciones de políticas son estudiadas en los modelos econométricos mediante la incorporación de ciertas variables de política en las ecuaciones del sistema, de tal modo que al modificar éstas de un modo específico puede observarse la reacción del sistema completo. Se requieren dos simulaciones básicas: una como punto de referencia o solución de control y una solución de perturbaciones, que en esencia constituye la misma solución de control más el cambio en la variable de política cuyos efectos estamos interesados en conocer.

${ }^{8}$ Para una descripción sobre la estructura del modelo véase R. Montemayor, op. cit., cap. IV, y A. Beltran del Rio. op. cit., cap. V. 
Aún cuando se pueden realizar muchas otras clases de estudios de simulación, ${ }^{9}$ estamos interesados en la respuesta dinámica del sistema ante diferentes acciones de política. Un modo de observar estas respuestas es computar un conjunto de multiplicadores dinámicos para cada política considerada. Por lo general estos multiplicadores se calculan como sigue:

$$
M=\frac{Y_{t}^{d}-Y_{t}}{\Delta P V_{t}}
$$

en donde:

$$
\begin{aligned}
& Y_{t}^{d}=\text { valor de la perturbación de } Y \\
& Y_{t}=\text { punto de referencia o de control de } Y \\
& \Delta P V_{t}=\text { cambio en la variable de política. }
\end{aligned}
$$

Esto es, el cambio en las variables endógenas $(Y)$, dividido por el monto del cambio en la variable de política bajo consideración, nos dará una estimación del multiplicador para la variable $Y$. Sin embargo, dada la naturaleza no lineal del modelo y la presencia de retardos, estamos en posibilidad, para algunos períodos, de tolerar la influencia de la mayoría de los retardos que han sufrido su mayor efecto para tener una idea de los valores de "equilibrio" o de "largo plazo" de los multiplicadores. Sin embargo, dado qua estamos tratando con un sistema complejo, dinámico, de ecuaciones de diferencias que es comparable a disponer de raíces que producen respuestas fluctuantes ante cambios en las fuerzas de que se derivan (exógenas), la tendencia en el tiempo de los multiplicadores es probable que fluctúe. No obstante, estos ejercicios pueden ser útiles para determinar los efectos relativos de acciones alternativas de política.

En principio, podríamos cambiar cualquiera de las variables predeterminadas del sistema y calcular multiplicadores que muestren efectos sobre el sistema. Sin embargo, limitaremos nuestro examen a las políticas que afectan las funciones de producción de la economía como están representadas en la matriz de insumo-producto, o sea sobre lo que sucedería si cambia el gasto del gobierno digamos en cien mil millones de pesos y el incremento del gasto se aplica a mejorar la eficiencia de un sector específico de la economía. No sólc existen cambios globales, estos a su vez conducen a cambios en la estructura productiva de la economía. Preguntas de esta clase pueden responderse, por lo menos parcialmente, mediante el uso de matrices de insumo-producto.

- Véase L.R. Klein, An Essay on the Theary of Economic Prediction, Chicago, Markham Publishing Co., 1971; también véase G. Fromm y P. Taubman, Policy Simulations with an Econometric Model, Washington, D.C. The Brookings Institution, 1968 , pp. $23-51$. 
En esencia existen dos caminos por los que podemos tratar este problema. Por una parte, puede suponerse que como resultado de un cambio de política un sector dado resulta más eficiente (tal vez por la importación de mejor equipo), de tal modo que requiere menor producción de otros sectores por unidad de su propia producción. En forma alternativa, puede decirse que si el mismo sector es capaz de entregar insumos de mejor calidad a los otros sectores, éstos necesitan menos insumos de tal sector por unidad de producción.

El primer caso significa un cambio en la función-producción del sector en cuestión, o sea que han cambiado las columnas de los sectores en la matriz de requerimientos directos.

En términos de coeficientes técnicos, los cambios significan una reducción en la columna de coeficientes que pertenecen al sector.

El segundo caso es equivalente al cambio en el renglón de coeficientes de la matriz $A$, el renglón que pertenece al sector que entrega los mejores insumos como resultado de la política. El caso puede ser considerado como un cambio en las funciones de producción de todos los sectores, un cambio que pertenece sólo al uso de la producción de un sector específico.

El modelo mexicano de insumo-producto está compuesto de 15 sectores, ${ }^{10}$ tres de los cuales se eligieron para efectuar este tipo de cálculos. Se seleccionó un sector por cada uno de los tres principales sectores de la economía (primario, secundario y terciario):

1: agricultura (sector primario)

2: industria de metales básicos (sector secundario)

3: transportes (sector terciario).

Se seleccionaron estos sectores por su importancia en las economías en desarrollo. La industria de metales básicos, que incluye industrias pesadas como la del acero es usualmente considerada como un sector clave en el desarrollo. Es común, en economías en desarrollo, que los gobiernos realicen esfuerzos desmedidos para promover la industria pesada, muchas veces en detrimento de la agricultura.

Se efectuaron dos procesos de simulación para cada uno de los sectores señalados. En una de ellas se consideró como supuesto que el incremento en la inversión del gobierno se agregó a una reducción del $10 \%$ en la columna de coeficientes del sector. En la otra se supone el mismo cambio en la inversión del gobierno pero ahora el incremento en la eficiencia se refleja en los renglones de coeficientes. De nuevo se supone una reducción de $10 \%$ en los renglones de coeficientes.

En todos les seis casos, los cambios se hicieron en la matriz $A$. Esto implica que la matriz $C$ de conversión de producción ha sido recalculada. Recordemos que:

$$
C=B(I-A)^{-1} H
$$

${ }^{10}$ La lista de 15 sectores aparece en la página 388. 
Aquí suponemos que la distribución industrial de la demanda final, la matriz $H$, permanece constante. Las otras dos matrices, $B$ e $(I-A)^{-1}$, cambian como resultado de los cambios en $A$. Una vez que la nueva matriz $C$ es recalculada, se probó un modelo para un período de seis años, a partir de 1961.

En este caso se obtuvo una solución de control al utilizar valores históricos para todas las variables exógenas. La solución alterada incluye dos cambios: el incremento de 1000 millones de pesos en la inversión real del gobierno y el cambio en los coeficientes de la matriz $A$. Se hizo también una simulación para calcular los efectos de un cambio sólo en los gastos del gobierno. A esta simulación le llamaremos Política 1 (véase el cuadro 1).

Cuadro 1

ECUACIONES DE ERROR ${ }^{a}$

\begin{tabular}{|c|c|c|c|c|c|c|c|}
\hline & & Constante & PC1-1 & PC2-1 & $\mathrm{PC}_{3}-1$ & $\mathrm{R}^{2}$ & $\mathrm{DN}$ \\
\hline Brror & & $\begin{array}{c}0.369 \\
(1.085)\end{array}$ & $\begin{array}{c}3.969 \\
(11.828)\end{array}$ & $\begin{array}{c}1.636 \\
(1.622)\end{array}$ & $\begin{array}{c}2.582 \\
(2.509)\end{array}$ & 0.95 & 2.91 \\
\hline Brror & 2 & $\begin{array}{c}-0.056 \\
(-1.063)\end{array}$ & $\begin{array}{c}0.459 \\
(8.825)\end{array}$ & $\begin{array}{c}-0.078 \\
(-0.495)\end{array}$ & $\begin{array}{c}0.192 \\
(1.159)\end{array}$ & 0.93 & 1.80 \\
\hline Error & 3 & $\begin{array}{c}0.499 \\
(4.995)\end{array}$ & $\begin{array}{c}-1.178 \\
(-11.936)\end{array}$ & $\begin{array}{c}0.178 \\
(0.599)\end{array}$ & $\begin{array}{l}-0.204 \\
(-0.648)\end{array}$ & 0.96 & 2.33 \\
\hline Error & 4 & $\begin{array}{c}0.288 \\
(2.350)\end{array}$ & $\begin{array}{l}-0.227 \\
(-1.841)\end{array}$ & $\begin{array}{c}0.975 \\
(2.683)\end{array}$ & $\begin{array}{l}-0.277 \\
(-0.718)\end{array}$ & 0.74 & 2.72 \\
\hline Error & 5 & $\begin{array}{l}-0.045 \\
(-0.366)\end{array}$ & $\begin{array}{c}-0.858 \\
(-7.069)\end{array}$ & $\begin{array}{c}-0.019 \\
(-0.051)\end{array}$ & $\begin{array}{c}0.015 \\
(0.038)\end{array}$ & 0.89 & 2.61 \\
\hline Error & 6 & $\begin{array}{l}0.192 \\
(1.983)\end{array}$ & $\begin{array}{c}0.029 \\
(1.805)\end{array}$ & $\begin{array}{c}0.464 \\
(1.617)\end{array}$ & $\begin{array}{c}0.161 \\
(0.528)\end{array}$ & 0.70 & 1.44 \\
\hline Brror & 7 & $\begin{array}{l}-0.007 \\
(-0.077)\end{array}$ & $\begin{array}{c}-1.512 \\
(17.508)\end{array}$ & $\begin{array}{c}-0.063 \\
(-0.244)\end{array}$ & $\begin{array}{c}-0.430 \\
(-1.564)\end{array}$ & 0.98 & 2.37 \\
\hline Error & 8 & $\begin{array}{l}-0.006 \\
(-0.141)\end{array}$ & $\begin{array}{l}-0.166 \\
(-4.157)\end{array}$ & $\begin{array}{l}-0.050 \\
(-0.417)\end{array}$ & $\begin{array}{c}0.024 \\
(0.185)\end{array}$ & 0.69 & 2.97 \\
\hline Error & 9 & $\begin{array}{c}0.143 \\
(2.981)\end{array}$ & $\begin{array}{c}-0.225 \\
(-4.740)\end{array}$ & $\begin{array}{c}0.184 \\
(1.287)\end{array}$ & $\begin{array}{c}0.179 \\
(1.182)\end{array}$ & 0.86 & 1.40 \\
\hline Error & 10 & $\begin{array}{c}0.543 \\
(2.198)\end{array}$ & $\begin{array}{l}-2.063 \\
(-8.462)\end{array}$ & $\begin{array}{l}0.836 \\
(1.141)\end{array}$ & $\begin{array}{c}0.702 \\
(1.904)\end{array}$ & 0.93 & 1.62 \\
\hline Error & 11 & $\begin{array}{l}0.341 \\
(1.457)\end{array}$ & $\begin{array}{c}0.877 \\
(3.803)\end{array}$ & $\begin{array}{c}0.216 \\
(1.313)\end{array}$ & $\begin{array}{c}0.492 \\
(0.670)\end{array}$ & 0.65 & 1.98 \\
\hline Error & 12 & $\begin{array}{l}0.083 \\
(1.342)\end{array}$ & $\begin{array}{r}-1.108 \\
-18.072\end{array}$ & $\begin{array}{c}-0.203 \\
(-1.105)\end{array}$ & $\begin{array}{c}-0.279 \\
(-1.431)\end{array}$ & 0.98 & 2.01 \\
\hline Error & 13 & $\begin{array}{c}1.790 \\
(3.557)\end{array}$ & $\begin{array}{c}-2.712 \\
(-5.463)\end{array}$ & $\begin{array}{c}2.540 \\
(1.703)\end{array}$ & $\begin{array}{l}1.227 \\
(0.776)\end{array}$ & 0.90 & 1.33 \\
\hline Error & 14 & $\begin{array}{c}0.058 \\
(1.220)\end{array}$ & $\begin{array}{l}-0.209 \\
(-5.656)\end{array}$ & $\begin{array}{c}-0.381 \\
(-2.662)\end{array}$ & $\begin{array}{c}-0.093 \\
(-0.610)\end{array}$ & 0.75 & 2.14 \\
\hline Error & 15 & $\begin{array}{c}10.484 \\
(39.488)\end{array}$ & $\begin{array}{c}-3.568 \\
(-13.522)\end{array}$ & $\begin{array}{c}0.439 \\
(0.553)\end{array}$ & $\begin{array}{c}1.010 \\
(1.203)\end{array}$ & 0.97 & 1.31 \\
\hline
\end{tabular}

a Los valores entre paréntesis son los valores de $t$.

- Prueba Durwin Watson.

En los cuadros 2 a 7 se muestran los efectos de cada caso sobre la producción de los 15 sectores, sobre los tres principales sectores agrupados y el producto total.

El primer aspecto que se observa es que un cambio en una columna, en la función-producción del sector da lugar a un incremento sustancial en la producción de dicho sector. Para apreciar mejor este cambio, 
Cuadro 2

Multiplicadores del Producto SECTORIAL: POLÍtica $1^{\text {a }}$ (miles de millones de pesos de 1950)

\begin{tabular}{|c|c|c|c|c|c|c|}
\hline \multirow[b]{2}{*}{ Sectores } & \multicolumn{6}{|c|}{ Pertodos } \\
\hline & 9 & 2 & 3 & 4 & 5 & 6 \\
\hline $\begin{array}{r}1 \\
2 \\
3 \\
4 \\
5 \\
6 \\
7 \\
8 \\
9 \\
10 \\
11 \\
12 \\
13 \\
14 \\
15\end{array}$ & $\begin{array}{l}0.11 \\
0.01 \\
0.04 \\
0.03 \\
0.03 \\
0.03 \\
0.03 \\
0.03 \\
0.04 \\
0.08 \\
0.22 \\
0.01 \\
0.32 \\
0.02 \\
0.09\end{array}$ & $\begin{array}{l}0.21 \\
0.00 \\
0.07 \\
0.06 \\
0.05 \\
0.05 \\
0.05 \\
0.05 \\
0.07 \\
0.13 \\
0.32 \\
0.02 \\
0.56 \\
0.05 \\
0.16\end{array}$ & $\begin{array}{l}0.28 \\
0.00 \\
0.09 \\
0.09 \\
0.07 \\
0.06 \\
0.06 \\
0.06 \\
0.08 \\
0.16 \\
0.40 \\
0.03 \\
0.77 \\
0.07 \\
0.24\end{array}$ & $\begin{array}{l}0.33 \\
0.00 \\
0.10 \\
0.11 \\
0.08 \\
0.06 \\
0.07 \\
0.06 \\
0.09 \\
0.17 \\
0.43 \\
0.04 \\
0.91 \\
0.08 \\
0.30\end{array}$ & $\begin{array}{r}0.38 \\
-0.01 \\
0.12 \\
0.14 \\
0.10 \\
0.07 \\
0.08 \\
0.07 \\
0.10 \\
0.19 \\
0.49 \\
0.04 \\
1.09 \\
0.10 \\
0.36\end{array}$ & $\begin{array}{r}0.29 \\
-0.04 \\
0.09 \\
0.11 \\
0.08 \\
0.06 \\
0.06 \\
0.05 \\
0.07 \\
0.15 \\
0.38 \\
0.03 \\
0.93 \\
0.09 \\
0.30\end{array}$ \\
\hline
\end{tabular}

" Mil millones de pesos de incremento en la inversión real del gobierno, sin cambio en las pautas de financiamiento público

\section{Cuadro 3}

MUltiplicadores: inCREMENTOS DE MIL Millones DE PESOS EN LA INVERSIÓN REAL DEL GOBIERNO Y DE $10 \%$ EN LA EFICIENCIA DEL SECTOR AGRÍCOLA

(cambios de columna en la matriz A)

\begin{tabular}{|c|c|c|c|c|c|c|}
\hline \multirow[t]{2}{*}{ Soctore: } & \multicolumn{6}{|c|}{ Pexiodos } \\
\hline & 1 & 2 & 3 & 4 & 5 & 6 \\
\hline $\begin{array}{r}1 \\
2 \\
3 \\
4 \\
5 \\
6 \\
7 \\
8 \\
9 \\
10 \\
11 \\
12 \\
13 \\
14 \\
15\end{array}$ & $\begin{array}{l}0.37 \\
0.01 \\
0.00 \\
0.01 \\
0.01 \\
0.02 \\
0.00 \\
0.03 \\
0.04 \\
0.07 \\
0.21 \\
0.01 \\
0.24 \\
0.02 \\
0.07\end{array}$ & $\begin{array}{l}0.46 \\
0.00 \\
0.03 \\
0.02 \\
0.03 \\
0.04 \\
0.02 \\
0.05 \\
0.06 \\
0.12 \\
0.31 \\
0.01 \\
0.47 \\
0.05 \\
0.14\end{array}$ & $\begin{array}{l}0.57 \\
0.00 \\
0.05 \\
0.04 \\
0.05 \\
0.05 \\
0.03 \\
0.06 \\
0.07 \\
0.15 \\
0.39 \\
0.02 \\
0.67 \\
0.06 \\
0.22\end{array}$ & $\begin{array}{l}0.63 \\
0.00 \\
0.06 \\
0.06 \\
0.06 \\
0.05 \\
0.02 \\
0.06 \\
0.08 \\
0.15 \\
0.41 \\
0.03 \\
0.79 \\
0.07\end{array}$ & $\begin{array}{l}0.70 \\
0.01 \\
0.07 \\
0.08 \\
0.08 \\
0.06 \\
0.03 \\
0.07 \\
0.09 \\
0.17 \\
0.47 \\
0.03 \\
0.96 \\
0.09 \\
0.33\end{array}$ & $\begin{array}{l}0.66 \\
0.04 \\
0.05 \\
0.05 \\
0.05 \\
0.05 \\
0.02 \\
0.05 \\
0.06 \\
0.13 \\
0.36 \\
0.02 \\
0.79 \\
0.08 \\
0.26\end{array}$ \\
\hline $\begin{array}{l}\text { Prianirio } \\
\text { Seotundario } \\
\text { Teroiario } \\
\text { Total }\end{array}$ & $\begin{array}{l}0.37 \\
0.39 \\
0.33 \\
1.09\end{array}$ & $\begin{array}{l}0.48 \\
0.69 \\
0.66 \\
1.83\end{array}$ & $\begin{array}{l}0.57 \\
0.91 \\
0.95 \\
2.43\end{array}$ & $\begin{array}{l}0.63 \\
0.98 \\
1.13 \\
2.74\end{array}$ & $\begin{array}{l}0.70 \\
1.14 \\
1.38 \\
3.22\end{array}$ & $\begin{array}{l}0.66 \\
0.80 \\
1.13 \\
2.59\end{array}$ \\
\hline
\end{tabular}


Cuadro 4

MUltiplicadores: inCREMENTOS DE MiL MILlones DE PESOS EN LA INVERSión REAL DEL GOBIERNO Y DE $10 \%$ EN LA EFICIENCIA DE LA INDUSTRIA DE METALES BÁSICOS

(cambios de columna en la matriz A)

\begin{tabular}{|c|c|c|c|c|c|c|}
\hline \multirow[t]{2}{*}{ Sectore: } & \multicolumn{6}{|c|}{ Periodos } \\
\hline & 1 & 2 & 3 & 4 & 5 & 6 \\
\hline $\begin{array}{r}1 \\
2 \\
3 \\
4 \\
5 \\
6 \\
7 \\
8 \\
9 \\
10 \\
11 \\
12 \\
13 \\
14 \\
15\end{array}$ & $\begin{array}{l}0.11 \\
0.01 \\
0.02 \\
0.03 \\
0.03 \\
0.03 \\
0.03 \\
0.03 \\
0.13 \\
0.08 \\
0.22 \\
0.01 \\
0.30 \\
0.02 \\
0.07\end{array}$ & $\begin{array}{l}0.21 \\
0.02 \\
0.05 \\
0.06 \\
0.05 \\
0.05 \\
0.05 \\
0.05 \\
0.17 \\
0.13 \\
0.32 \\
0.02 \\
0.54 \\
0.05 \\
0.14\end{array}$ & $\begin{array}{l}0.28 \\
0.02 \\
0.07 \\
0.09 \\
0.07 \\
0.06 \\
0.06 \\
0.06 \\
0.20 \\
0.16 \\
0.40 \\
0.03 \\
0.75 \\
0.07 \\
0.22\end{array}$ & $\begin{array}{l}0.33 \\
0.03 \\
0.08 \\
0.11 \\
0.08 \\
0.06 \\
0.07 \\
0.06 \\
0.22 \\
0.17 \\
0.43 \\
0.03 \\
0.88 \\
0.08 \\
0.28\end{array}$ & $\begin{array}{l}0.38 \\
0.04 \\
0.09 \\
0.14 \\
0.10 \\
0.07 \\
0.08 \\
0.07 \\
0.25 \\
0.19 \\
0.49 \\
0.93 \\
1.00 \\
0.10 \\
0.34\end{array}$ & $\begin{array}{l}0.29 \\
0.08 \\
0.06 \\
0.11 \\
0.08 \\
0.06 \\
0.06 \\
0.05 \\
0.24 \\
0.15 \\
0.38 \\
0.02 \\
0.00 \\
0.00 \\
0.28\end{array}$ \\
\hline $\begin{array}{l}\text { Primario } \\
\text { Seoundasio } \\
\text { Perolario } \\
\text { Total }\end{array}$ & $\begin{array}{l}0.11 \\
0.59 \\
0.39 \\
1.09\end{array}$ & $\begin{array}{l}0.21 \\
0.93 \\
0.73 \\
1.87\end{array}$ & $\begin{array}{l}0.28 \\
1.18 \\
1.04 \\
2.50\end{array}$ & $\begin{array}{l}0.33 \\
1.25 \\
1.24 \\
2.82\end{array}$ & $\begin{array}{l}0.38 \\
1.4\end{array}$ & $\begin{array}{l}0.29 \\
1.12 \\
1.2\end{array}$ \\
\hline
\end{tabular}

Cuadro 5

Multiplicadores: inCREMENTos DE MIL MILlones de PESOS EN LA INVERSión REAL DEL GOBIERNO Y DEL 10\% EN LA EFICIENCIA DEL SECTOR TRANSPORTE (cambios de columna en la matriz A)

\begin{tabular}{|c|c|c|c|c|c|c|}
\hline \multirow{2}{*}{ Seotorea } & \multicolumn{6}{|c|}{ Periodoe } \\
\hline & 1 & 2 & 3 & 4 & 5 & 6 \\
\hline $\begin{array}{r}1 \\
2 \\
3 \\
4 \\
5 \\
6 \\
7 \\
8 \\
9 \\
10 \\
11 \\
12 \\
13 \\
14 \\
15\end{array}$ & $\begin{array}{l}0.11 \\
0.01 \\
0.01 \\
0.03 \\
0.03 \\
0.03 \\
0.02 \\
0.03 \\
0.04 \\
0.08 \\
0.22 \\
0.01 \\
0.29 \\
0.14 \\
0.07\end{array}$ & $\begin{array}{l}0.29 \\
0.00 \\
0.02 \\
0.06 \\
0.05 \\
0.05 \\
0.04 \\
0.05 \\
0.07 \\
0.13 \\
0.32 \\
0.02 \\
0.52 \\
0.18 \\
0.14\end{array}$ & $\begin{array}{l}0.28 \\
0.00 \\
0.04 \\
0.09 \\
0.07 \\
0.06 \\
0.05 \\
0.06 \\
0.08 \\
0.15 \\
0.40 \\
0.03 \\
0.73 \\
0.21 \\
0.22\end{array}$ & $\begin{array}{l}0.33 \\
0.00 \\
0.04 \\
0.11 \\
0.08 \\
0.06 \\
0.06 \\
0.06 \\
0.09 \\
0.16 \\
0.42 \\
0.04 \\
0.86 \\
0.23 \\
0.28\end{array}$ & $\begin{array}{l}0.37 \\
0.01 \\
0.06 \\
0.14 \\
0.10 \\
0.07 \\
0.07 \\
0.07 \\
0.10 \\
0.18 \\
0.48 \\
0.04 \\
1.04 \\
0.26 \\
0.34\end{array}$ & $\begin{array}{l}0.28 \\
0.04 \\
0.02 \\
0.11 \\
0.08 \\
0.06 \\
0.04 \\
0.05 \\
0.07 \\
0.14 \\
0.37 \\
0.03 \\
0.87 \\
0.26 \\
0.27\end{array}$ \\
\hline $\begin{array}{l}\text { Primario } \\
\text { Seoundario } \\
\text { Terolario } \\
\text { Total }\end{array}$ & $\begin{array}{l}0.11 \\
0.49 \\
0.50 \\
1.10\end{array}$ & $\begin{array}{l}0.29 \\
0.81 \\
0.84 \\
1.86\end{array}$ & $\begin{array}{l}0.28 \\
1.03 \\
1.16 \\
2.47\end{array}$ & $\begin{array}{l}0.33 \\
1.12 \\
1.37 \\
2.82\end{array}$ & $\begin{array}{l}0.37 \\
1.30 \\
1.64 \\
3.31\end{array}$ & $\begin{array}{l}0.28 \\
0.93 \\
1.40 \\
2.61\end{array}$ \\
\hline
\end{tabular}


compárense estos resultados con los obtenidos para un incremento sólo en la inversión del gobierno( Política 1). Así, el cambio en la columna de coeficientes de la agricultura tiene un multiplicador en la producción de dicho sector de alrededor de 0.32 miles de millones de pesos (a precios de 1950) al final de los cinco períodos, mayor que el multiplicador de la Política 1 (véase el cuadro 1). Esta diferencia es casi la misma que la del multiplicador de la Política 1 para cada sector $(0.38)$.

Si se cambia la función-producción de la industria de metales básicos, el multiplicador para este sector, al final de los cinco períodos, es más que el doble del multiplicador de la Política 1 para el mismo sector.

Para el cambio en la columna del sector transporte, el multiplicador es 0.26 miles de millones de pesos (de 1950), comparado con un multiplicador de 0.10 con la Política 1.

En cada uno de los cambios en las columnas, se observa que un sector resulta más eficiente en cuanto al uso de la producción de los otros sectores. Así, si comparamos sus efectos (multiplicadores) con los de la Política 1 , se encuentra que para estos sectores los efectos de la Política 1 son mayores en la mayoría de los casos.

Un cambio en la columna de la función-producción de la agricultura origina que todos los sectores, excepto la minería y los sectores de metales no ferrosos, registren multiplicadores más pequeños que cuando solo se incrementa la inversión del gobierno (Política 1). Lo anterior puede apreciarse en el cuadro 2 en el que se muestra que un cambio en la columna de la agriçultura genera un multiplicador más pequeño que la Política 1 para los sectores secundario y terciario. Un incremento en la eficiencia del sector agrícola tiene como efecto reducir comparativamente la demanda de productos de sus principales abastecedores. Así, dado un incremento en la eficiencia, se requieren menos productos de otros sectores para satisfacer un nivel determinado de demanda final. En cierto sentido, significa que algunos recursos son liberados por efecto del cambio, los recursos que pueden ser utilizados para otros propósitos permiten un incremento adicional en la actividad económica.

Las industrias de metales básicos tienen un menor efecto propagador. Sólo los sectores 2, 3, 13 y 15 se ven afectádos por este cambio en la función-producción.

Cuando cambia la columna del sector de transportes, el efecto se resiente principalmente en los sectores $3,7,10,13$ y 15 . Ninguno de estos sectores registra un efecto apreciable sobre la agricultura. Los cambios en la columna de transportes tienen sólo un efecto reducido sobre la agricultura. En resumen, se observa que un cambio en las funcionesproducción sectoriales tiende a modificar la composición del producto con ganancias relativas a su favor y pérdidas relativas para sus principales abastecedores.

El segundo conjunto de simulaciones que supone una reducción de los renglones de coeficientes de la matriz $A$, se presenta en los cuadros 6 a 8. Este caso, 'como se mencionó antes, es equivalente a un cambio en 
Cuadro 6

Multiplicadores: INCREMENTTOS DE MIL MILLONES DE PESOS EN LA INVERSIón REAL DEL GOBIERNO Y DEL $10 \%$ EN LA EFICIENCIA DEL SECTOR AGRÍCOLA (cambios de renglón en la matriz $A$ )

\begin{tabular}{ccccccc}
\hline \multirow{2}{*}{ Seotores } & \multicolumn{7}{c}{ Periodos } \\
\cline { 2 - 7 } & \multicolumn{1}{c}{1} & 2 & 3 & 4 & 5 & 6 \\
\hline 1 & 0.30 & 0.21 & 0.16 & 0.14 & 0.11 & 0.23 \\
2 & 0.01 & 0.00 & 0.00 & 0.00 & 0.01 & 0.04 \\
3 & 0.03 & 0.06 & 0.09 & 0.10 & 0.12 & 0.09 \\
4 & 0.42 & 0.47 & 0.52 & 0.57 & 0.63 & 0.64 \\
5 & 0.08 & 0.10 & 0.13 & 0.14 & 0.17 & 0.15 \\
6 & 0.05 & 0.08 & 0.09 & 0.09 & 0.11 & 0.10 \\
7 & 0.05 & 0.07 & 0.08 & 0.10 & 0.11 & 0.10 \\
8 & 0.03 & 0.05 & 0.06 & 0.06 & 0.07 & 0.05 \\
9 & 0.04 & 0.07 & 0.08 & 0.09 & 0.10 & 0.07 \\
10 & 0.08 & 0.14 & 0.17 & 0.18 & 0.19 & 0.16 \\
11 & 0.23 & 0.33 & 0.41 & 0.45 & 0.52 & 0.44 \\
12 & 0.01 & 0.02 & 0.03 & 0.04 & 0.04 & 0.04 \\
13 & 0.32 & 0.55 & 0.77 & 0.92 & 1.12 & 0.99 \\
14 & 0.02 & 0.06 & 0.07 & 0.08 & 0.10 & 0.10 \\
15 & 0.09 & 0.16 & 0.25 & 0.31 & 0.38 & 0.35 \\
Primario & -0.30 & -0.21 & -0.16 & -0.14 & -0.11 & -0.23 \\
Sooundario & 1.03 & 1.39 & 1.66 & 1.82 & 2.05 & 1.80 \\
Teroiario & 0.43 & 0.77 & 1.09 & 1.31 & 1.70 & 1.44 \\
Total & 1.16 & 1.95 & 2.59 & 2.99 & 3.64 & 3.01 \\
\hline
\end{tabular}

Cuadro 7

MUlTiPliCadores: INCREMENTOS DE MIL MILLONES DE PESOS EN LA INVERSIón REAL DEL GOBIERNO Y DEL $10 \%$ EN LA EFICIENCIA EN LAS INDUSTRIAS DE METALES BÁSICOS

(cambios de renglón en la matriz $A$ )

\begin{tabular}{ccccccc}
\hline \multirow{2}{*}{ Sootores } & \multicolumn{7}{c}{ Periodos } \\
\cline { 2 - 7 } & 1 & 2 & 3 & 4 & 5 & 6 \\
\hline 1 & 0.11 & 0.21 & 0.28 & 0.33 & 0.38 & 0.29 \\
2 & 0.00 & 0.01 & 0.02 & 0.02 & 0.03 & 0.06 \\
3 & 0.03 & 0.06 & 0.07 & 0.08 & 0.10 & 0.07 \\
4 & 0.04 & 0.07 & 0.10 & 0.12 & 0.15 & 0.12 \\
5 & 0.03 & 0.05 & 0.07 & 0.08 & 0.10 & 0.08 \\
6 & 0.03 & 0.05 & 0.06 & 0.06 & 0.07 & 0.06 \\
7 & 0.03 & 0.05 & 0.06 & 0.07 & 0.08 & 0.06 \\
8 & 0.03 & 0.05 & 0.06 & 0.06 & 0.07 & 0.05 \\
9 & -0.03 & -0.01 & -0.01 & -0.01 & -0.01 & -0.06 \\
10 & 0.14 & 0.19 & 0.23 & 0.25 & 0.28 & 0.29 \\
11 & 0.27 & 0.38 & 0.47 & 0.52 & 0.59 & 0.52 \\
12 & 0.01 & 0.02 & 0.03 & 0.04 & 0.04 & 0.03 \\
13 & 0.31 & 0.55 & 0.76 & 0.89 & 1.07 & 0.91 \\
14 & 0.02 & 0.05 & 0.07 & 0.08 & 0.10 & 0.09 \\
15 & 0.08 & 0.15 & 0.23 & 0.29 & 0.35 & 0.29 \\
Primario & 0.11 & 0.21 & 0.28 & 0.33 & 0.38 & 0.27 \\
Seoundario & 0.58 & 0.90 & 1.12 & 1.25 & 1.44 & 1.16 \\
Torolario & 0.41 & 0.75 & 1.03 & 1.26 & 1.52 & 1.29 \\
Total & 1.10 & 1.86 & 2.43 & 2.84 & 3.34 & 2.74 \\
\hline
\end{tabular}




\section{Cuadro 8}

MULTIPLICADORES: INCREMENTOS DE MIL MILLONES DE PESOS EN LA INVERSIÓN REAL DEL GOBIERNO Y DEL $10 \%$ EN LA EFICIENCIA DEL TRANSPORTE (cambios de renglón en la matriz $A$ )

\begin{tabular}{ccccccr}
\hline \multirow{2}{*}{ Sootores } & \multicolumn{7}{c}{ Periodoe } \\
\cline { 2 - 7 } & 1 & 2 & 3 & 4 & 5 & \multicolumn{1}{c}{} \\
\hline 1 & 0.11 & 0.21 & 0.28 & 0.33 & 0.38 & 0.29 \\
2 & 0.01 & 0.00 & 0.00 & 0.00 & -0.01 & -0.04 \\
3 & 0.02 & 0.05 & 0.07 & 0.08 & 0.10 & 0.07 \\
4 & 0.03 & 0.06 & 0.09 & 0.11 & 0.14 & 0.11 \\
5 & 0.03 & 0.05 & 0.07 & 0.08 & 0.10 & 0.08 \\
6 & 0.03 & 0.05 & 0.06 & 0.06 & 0.07 & 0.06 \\
7 & 0.03 & 0.05 & 0.06 & 0.07 & 0.08 & 0.06 \\
8 & 0.03 & 0.05 & 0.06 & 0.06 & 0.07 & 0.05 \\
9 & 0.04 & 0.07 & 0.08 & 0.09 & 0.10 & 0.07 \\
10 & 0.08 & 0.13 & 0.16 & 0.17 & 0.19 & 0.16 \\
11 & 0.22 & 0.32 & 0.40 & 0.43 & 0.49 & 0.38 \\
12 & 0.01 & 0.02 & 0.03 & 0.04 & 0.04 & 0.03 \\
13 & 0.45 & 0.70 & 0.32 & 1.07 & 1.26 & 1.16 \\
14 & -0.09 & -0.07 & -0.06 & -0.06 & -0.05 & -0.06 \\
15 & 0.09 & 0.16 & 0.24 & 0.30 & 0.36 & 0.30 \\
Primario & 0.11 & 0.21 & 0.38 & 0.33 & 0.38 & 0.29 \\
Sooundario & 0.52 & 0.85 & 1.08 & 1.19 & 1.37 & 1.03 \\
Toroiario & 0.45 & 0.79 & 1.10 & 1.31 & 1.57 & 1.40 \\
Total & 1.08 & 1.85 & 2.46 & 2.83 & 3.32 & 2.72 \\
\hline
\end{tabular}

las funciones de producción de los sectores que usan como insumos la producción del sector cuyo renglón se ha modificado.

Los resultados del cuadro 6 muestran que cuando suponemos un incremento en la eficiencia de la agricultura (reflejada ahora en un cambio en el renglón de coeficientes), el producto de la agricultura misma disminuye de manera sustancial. La función de producción de los otros sectores resulta ahora de tal forma que requieren menor producto de la agricultura por unidad de producción. Lo mismo se observa en los casos en que los renglones de las industrias de metales básicos y de transportes se reducen. Un hecho sorprendente es que el cambio en el renglón de agricultura produce multiplicadores más altos para los sectores secundario y terciario (véase el cuadro 9) y, a pesar de la reducción del producto de la agricultura, mayor estímulo al $P I B$. En este caso se produce un impulso mayor sobre el sector secundario o de manufacturas que en el caso de un cambio en la columna en las industrias de metales básicos.

Los resultados de la simulación anterior sugieren que los esfuerzos del desarrollo pueden ser favorables si se da mayor atención a la asignatura como sucede en algunos casos.

De manera usual, los gobiernos tienden a dar mayor énfasis al desarrollo del sector de industria pesada. Dado lo limitado de los recursos disponibles, esto significa que se desatiende el sector agrícola. Al mismo tiempo, se observa que esto podría constituir un mayor estímulo al desarrollo industrial si la agricultura fuera capaz de incrementar su productividad (mientras los precios de los productos agrícolas se mantengan 
Cuadro 9

SiMULACIÓN DE MULTIPLICADORES DE INSUMO PRODUCTO, COMPARADOS CON LOS DE LA POLÍTICA 1

\begin{tabular}{|c|c|c|c|c|}
\hline & \multicolumn{3}{|c|}{ Sectores } & \multirow[b]{2}{*}{ Total } \\
\hline & $I$ & II & III & \\
\hline \multirow{3}{*}{$\begin{array}{l}\text { S6lo invergión del gobierno (polftioa 1) } \\
\text { Cambio de renglón en la agricultura } \\
\text { Cambio de renglón en la indugtria } \\
\text { de metales basicos } \\
\text { Cambio de renglón en transportes } \\
\text { Cambio de columna en agricultura } \\
\text { Cambio de renglón en la indugtria } \\
\text { de metales básicos } \\
\text { Cambio de columna on transportes }\end{array}$} & $\begin{array}{r}0.38 \\
-0.11\end{array}$ & $\begin{array}{l}1.36 \\
2.05\end{array}$ & $\begin{array}{l}1.53 \\
1.70\end{array}$ & $\begin{array}{c}3.26 \\
3.64\end{array}$ \\
\hline & $\begin{array}{l}0.38 \\
0.38 \\
0.70\end{array}$ & $\begin{array}{l}1.44 \\
1.37 \\
1.14\end{array}$ & $\begin{array}{l}1.52 \\
1.57 \\
1.38\end{array}$ & $\begin{array}{l}3.34 \\
3.32 \\
3.22\end{array}$ \\
\hline & $\begin{array}{l}0.38 \\
0.37\end{array}$ & $\begin{array}{l}1.47 \\
1.30\end{array}$ & $\begin{array}{l}1.50 \\
1.64\end{array}$ & $\begin{array}{l}3.35 \\
3.31\end{array}$ \\
\hline
\end{tabular}

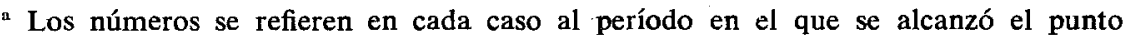
más elevado

b $\mathbf{I}=$ Primario

II $=$ Secundario

III $=$ Terciario

relativamente bajos) que cuando es relegada y se transforma en un "cuello de botella".

Los resultados anteriores están sujetos a una limitación. Recuérdese que:

y

$$
\left.Y_{i}=C G+U_{i} \quad(i=1 \ldots, 15) \quad 1\right)
$$$$
U_{i}=f\left(P C 1_{-1}, P C 2_{-1}, P C 3_{-1}\right)(i=1, \ldots, 15)
$$

Hemos visto que los errores de los modelos que tratamos de habilitar para los cambios en la matriz $C$, son función de los retados de las primeras tres componentes principales. A su vez, estas son combinaciones lineales de los errores. De este modo, si se introduce un cambio de política en el período $t, P C 1_{-1}, P C 2_{-1}$ y $P C 3_{-1}$ estarán determinados por los errores en $t$-1. Esto significa que la segunda parte del conjunto de ecuaciones (1) será independiente del cambio de política. Una manera de evitar este problema podría ser desarrollar las ecuaciones de las componentes principales. Esto es, hacer cada componente principal utilizada en (2) una función de alguna variable determinada en el modelo. En el Apéndice se presentan las gráficas de cada componente principal. En ellas se puede observar que la primera componente representa un factor de tendencia, la segunda un ciclo comercial estándar y la tercera un ciclo de corto plazo. Esto sugiere un camino posible para eliminar el problema anterior. ${ }^{11} \mathrm{Un}$ segundo problema con las simulaciones anteriores es que se ha supuesto que la matriz $H$, la distribución de la demanda final por industrias, se

11 Se ha realizado algún trabajo preliminar para implementar estas ideas, sin embargo, no se han obtenido resultados satisfactorios para la tercera componente principal que representa el ciclo de corto plazo. 
mantiene sin cambios. Un cambio en la matriz $H$ puede ser visualizado como un cambio en la composición de alguna categoría de demanda final. Por ejemplo, a medida que el tiempo pasa, puede haber un cambio en la composición del consumo. $O$ sea que los gastos en consumo total tuvieran una mayor proporción de bienes durables y menos productos alimenticios. Lo anterior significa, en términos de la matriz $H$, que las entregas de la agricultura y las industrias productoras de alimentos podrían representar una proporción reducida de las entregas totales al consumo, mientras que aquellos sectores que producen bienes durables como automóviles, refrigeradores, etc., podrían entregaran una mayor proporción. Se efectuó uno de tales cálculos cuyos resultados se muestran en el cuadro 10. Se estableció como supuesto que habría un incremento de $10 \%$ en la proporción de bienes durables en el consumo total y en concordancia una reducción similar en alimentos. Para lograr esto, se redujeron los coeficientes de entregas al consumo por parte de la agricultura y de la industria alimenticia y los del sector de fabricación de productos metálicos se incrementaron. Esto se refleja en el cuadro 10. Los sectores 1 y 4 (agricultura y alimentos) sufrieron una disminución en su producción debido a un cambio en la demanda. El sector 10 (fabricación y reparación de productos metálicos) muestra un incremento sustancial en su producción. Los efectos globales consistieron en un aumento en la producción total. Este significa que los moldes de cambio en la demanda producen un efecto positivo en la producción total. Sin embargo, el efecto más importante se verifica en la composición de la producción. El sector secundario adquiere importancia relativamente mayor y el sector primario disminuye.

\section{Cuadro 10}

EFectos de un CAMBIO EN LAS PAUTAS de CON SUMO: $10 \%$ DE INCREMENTO EN LA PROPORCIÓN DE BIENES DURABLES (indicadores seleccionados)

\begin{tabular}{|c|c|c|c|c|c|c|}
\hline \multirow{2}{*}{ Stotorell } & \multicolumn{6}{|c|}{ Periodo: } \\
\hline & 1 & 2 & 3 & 4 & 5 & 6 \\
\hline $\begin{array}{c}1 \\
2 \\
3 \\
4 \\
5 \\
6 \\
7 \\
8 \\
9 \\
10 \\
11 \\
12 \\
13 \\
14 \\
15 \\
\text { Primario } \\
\text { Sooundario } \\
\text { Torolario } \\
\text { Tatal }\end{array}$ & $\begin{array}{r}-3.10 \\
0.13 \\
0.04 \\
-1.38 \\
-0.01 \\
0.04 \\
0.11 \\
0.01 \\
0.42 \\
3.73 \\
0.01 \\
0.02 \\
0.00 \\
0.02 \\
0.14 \\
-3.10 \\
3.12 \\
0.16 \\
0.18\end{array}$ & $\begin{array}{r}-3.20 \\
0.14 \\
0.06 \\
-1.43 \\
0.00 \\
0.05 \\
0.12 \\
0.02 \\
0.45 \\
3.92 \\
0.05 \\
0.03 \\
0.07 \\
0.03 \\
0.17 \\
-3.20 \\
3.41 \\
0.27 \\
0.48\end{array}$ & $\begin{array}{r}-3.35 \\
0.15 \\
0.07 \\
-1.50 \\
0.01 \\
0.06 \\
0.14 \\
0.02 \\
0.48 \\
4.16 \\
0.10 \\
0.03 \\
0.16 \\
0.04 \\
0.21 \\
-3.35 \\
3.72 \\
0.41 \\
0.78\end{array}$ & $\begin{array}{r}-3.54 \\
0.17 \\
0.08 \\
-1.59 \\
0.02 \\
0.07 \\
0.15 \\
0.03 \\
0.53 \\
4.46 \\
0.17 \\
0.04 \\
0.27 \\
0.05 \\
0.27 \\
-3.54 \\
4.16 \\
0.59 \\
1.21\end{array}$ & $\begin{array}{r}-3.75 \\
0.18 \\
0.10 \\
-1.68 \\
0.03 \\
0.08 \\
0.17 \\
0.04 \\
0.57 \\
4.78 \\
0.22 \\
0.04 \\
0.39 \\
0.06 \\
0.33 \\
-3.75 \\
4.53 \\
1.78 \\
1.56\end{array}$ & $\begin{array}{r}-4.02 \\
0.20 \\
0.11 \\
-1.80 \\
0.04 \\
0.09 \\
0.19 \\
0.04 \\
0.61 \\
5.17 \\
0.23 \\
0.05 \\
0.48 \\
0.07 \\
0.40 \\
-4.02 \\
4.93 \\
0.95 \\
1.86\end{array}$ \\
\hline
\end{tabular}


Para concluir, debe tenerse en cuenta que aunque los resultados de los cuadros parecen concluyentes, están sujatos a cierto margen de error. No se dispone de estimaciones de los errores de predicción del modelo; sin embargo, el error estimado para un período de muestra señala que es razonable esperar un error de entre \pm 5 y $\pm 10 \%$ en la mayoría de los casos. De cualquier manera, los resultados pueden ser indicativos de los posibles efectos de diferentes acciones de política.

\section{RESUMEN}

El propósito del presente estudio fue mostrar el uso del análisis de insumo-producto en macromodelos. Esto se hizo mediante el ejemplo del caso mexicano.

Primero se desarrolló el algebra de la vinculación entre ambos modelos y se hicieron explícitas las diferentes identidades involucradas en el modelo de insumo-producto. Se presentaron algunos problemas en la vinculación, derivados de la disponibilidad de información y de los diferentes conceptos utilizados — brutos vs netos- en el sistema contable, pero se resolvieron con facilidad al establecer como supuesto una proporcionalidad similar con la matriz. $\boldsymbol{A}$. Esto a su vez resulta en el problema de cómo tratar el hecho de que los coeficientes cambian en el tiempo. Para esto se utilizaron las componentes principales para el modelo de errores o residuales generados por la utilización de coeficientes constantes.

Una vez establecido el vínculo entre el modelo de insumo-producto y el macromodelo, existen importantes acciones de política entre las cuales se puede elegir y que pueden utilizarse para efectuar simulaciones. Por ejemplo, ¿cómo puede ser modificada la estructura de la ecomía? ¿qué sectores se deben promover para lograr un crecimiento más rápido?, etc.

Se eligieron tres sectores para simular los efectos de la inversión del gobierno. Un hecho sorprendente que se derivó del ejercicio fue que la inversión en la agricultura, que tiende a incrementar su eficiencia (medida en el renglón de coeficientes) podría constituír un mayor estímulo para la producción industrial y -a pesar de una reducción en la producción agrícola misma - para la actividad total, que si la nueva inversión del gobierno se aplicara al sector industrial.

Los resultados, como se puntualizó antes, están sujetos a algunas limitaciones. De cualquier modo, sugieren nuevas direcciones en los esfuerzos para promover el desarrollo así como la utilidad de incorporar el análisis de insumo-producto en los modelos econométricos. 
A PEN D I C E

Cuadro A 1

Matriz $A$ DE REQUERIMIENTOS DIRECTOS. 1960

\begin{tabular}{|c|c|c|c|c|c|c|c|c|c|c|}
\hline Sectorea & 1 & 2 & 3 & 4 & 5 & 6 & 7 & 8 & 9 & 10 \\
\hline $\begin{array}{r}1 \\
2 \\
3 \\
4 \\
5 \\
6 \\
7 \\
8 \\
9 \\
10 \\
11 \\
12 \\
13 \\
14 \\
15\end{array}$ & $\begin{array}{l}0.06310 \\
0.0 \\
0.01930 \\
0.05160 \\
0.01620 \\
0.00380 \\
0.02300 \\
0.00010 \\
0.00150 \\
0.00700 \\
0.00440 \\
0.00230 \\
0.03730 \\
0.0 \\
0.00490\end{array}$ & $\begin{array}{l}0.00320 \\
0.23360 \\
0.03570 \\
0.00280 \\
0.00280 \\
0.00810 \\
0.02300 \\
0.00280 \\
0.01580 \\
0.01160 \\
0.00560 \\
0.02460 \\
0.03830 \\
0.00210 \\
0.03520\end{array}$ & $\begin{array}{l}0.00010 \\
0.01340 \\
0.30710 \\
0.00110 \\
0.00160 \\
0.00770 \\
0.00560 \\
0.00070 \\
0.00260 \\
0.00500 \\
0.00270 \\
0.00030 \\
0.02060 \\
0.00130 \\
0.02370\end{array}$ & $\begin{array}{l}0.27330 \\
0.00080 \\
0.01510 \\
0.15950 \\
0.00320 \\
0.01490 \\
0.00450 \\
0.00860 \\
0.00490 \\
0.01080 \\
0.00150 \\
0.00560 \\
0.08930 \\
0.00190 \\
0.02310\end{array}$ & $\begin{array}{l}0.08420 \\
0.00030 \\
0.01430 \\
0.00420 \\
0.19800 \\
0.00770 \\
0.05100 \\
0.00060 \\
0.00450 \\
0.00880 \\
0.00290 \\
0.00940 \\
0.11780 \\
0.00250 \\
0.02600\end{array}$ & $\begin{array}{l}0.09300 \\
0.00150 \\
0.01730 \\
0.00590 \\
0.00680 \\
0.00770 \\
0.02390 \\
0.00230 \\
0.00700 \\
0.00910 \\
0.00290 \\
0.01110 \\
0.08620 \\
0.00370 \\
0.04300\end{array}$ & $\begin{array}{l}0.06260 \\
0.01060 \\
0.01440 \\
0.01480 \\
0.01070 \\
0.02800 \\
0.11590 \\
0.00640 \\
0.00756 \\
0.01080 \\
0.00230 \\
0.00850 \\
0.09900 \\
0.00340 \\
0.03950\end{array}$ & $\begin{array}{l}0.00040 \\
0.04310 \\
0.08620 \\
0.00200 \\
0.00470 \\
0.03640 \\
0.01940 \\
0.07120 \\
0.01310 \\
0.01700 \\
0.00990 \\
0.02970 \\
0.09850 \\
0.00320 \\
0.03080\end{array}$ & $\begin{array}{l}0.00040 \\
0.09700 \\
0.05860 \\
0.00450 \\
0.00510 \\
0.00920 \\
0.00720 \\
0.01340 \\
0.1810 \\
0.01220 \\
0.09470 \\
0.01220 \\
0.06700 \\
0.00150 \\
0.05100\end{array}$ & $\begin{array}{l}0.00270 \\
0.001360 \\
0.01650 \\
0.00790 \\
0.00770 \\
0.01730 \\
0.04020 \\
0.00490 \\
0.11890 \\
0.08610 \\
0.00160 \\
0.00400 \\
0.05180 \\
0.00440 \\
0.02970\end{array}$ \\
\hline & 11 & 12 & 13 & 14 & 15 & & & & & \\
\hline $\begin{array}{r}1 \\
2 \\
3 \\
4 \\
5 \\
6 \\
7 \\
8 \\
9 \\
9 \\
10 \\
11 \\
12 \\
13 \\
14 \\
15 \\
\end{array}$ & $\begin{array}{l}0.00020 \\
0.01550 \\
0.00890 \\
0.00290 \\
0.00270 \\
0.04400 \\
0.01370 \\
0.10250 \\
0.08370 \\
0.06220 \\
0.00430 \\
0.00350 \\
0.11910 \\
0.00140 \\
0.02500 \\
\end{array}$ & $\begin{array}{l}0.0 \\
0.00220 \\
0.09790 \\
0.00090 \\
0.00230 \\
0.00590 \\
0.00500 \\
0.00050 \\
0.00590 \\
0.02360 \\
0.01950 \\
0.03630 \\
0.04220 \\
0.00540 \\
0.01950 \\
\end{array}$ & $\begin{array}{l}0.00010 \\
0.00020 \\
0.00300 \\
0.00100 \\
0.00170 \\
0.00800 \\
0.00150 \\
0.00040 \\
0.00250 \\
0.00380 \\
0.00180 \\
0.00470 \\
0.00650 \\
0.05490 \\
0.03010\end{array}$ & $\begin{array}{l}0.0 \\
0.00090 \\
0.12710 \\
0.00350 \\
0.00150 \\
0.00600 \\
0.03780 \\
0.00020 \\
0.00340 \\
0.01200 \\
0.00900 \\
0.00370 \\
0.07700 \\
0.00300 \\
0.03020\end{array}$ & $\begin{array}{l}0.00060 \\
0.00020 \\
0.00200 \\
0.00350 \\
0.00270 \\
0.01150 \\
0.01030 \\
0.00060 \\
0.00480 \\
0.01490 \\
0.01160 \\
0.00500 \\
0.04130 \\
0.00420 \\
0.10780\end{array}$ & & & & & \\
\hline
\end{tabular}


Cuadro A 2

MATRIZ INVERSA DE REQUERIMIENTOS DIRECTOS

\begin{tabular}{|c|c|c|c|c|c|c|c|c|c|c|}
\hline Seotores & 1 & 2 & 3 & 4 & 5 & 6 & 7 & 8 & 9 & 10 \\
\hline \multirow[t]{2}{*}{$\begin{array}{r}1 \\
2 \\
3 \\
4 \\
5 \\
6 \\
7 \\
8 \\
9 \\
10 \\
11 \\
12 \\
13 \\
14 \\
15\end{array}$} & $\begin{array}{l}1.09244 \\
0.00233 \\
0.03605 \\
0.00210 \\
0.02329 \\
0.00953 \\
0.03180 \\
0.00184 \\
0.00905 \\
0.01162 \\
0.00565 \\
0.00429 \\
0.05753 \\
0.00371 \\
0.01444\end{array}$ & $\begin{array}{l}0.01164 \\
1.31184 \\
0.08054 \\
0.00674 \\
0.00664 \\
0.01887 \\
0.03878 \\
0.00612 \\
0.03145 \\
0.02150 \\
0.00988 \\
0.03569 \\
0.05932 \\
0.00755 \\
0.00332\end{array}$ & $\begin{array}{l}0.00392 \\
0.02695 \\
1.44821 \\
0.00299 \\
0.00379 \\
0.01618 \\
0.01208 \\
0.00208 \\
0.00788 \\
0.01013 \\
0.00492 \\
0.00218 \\
0.03864 \\
0.00445 \\
0.04355\end{array}$ & $\begin{array}{l}0.35943 \\
0.00533 \\
0.04544 \\
1.21312 \\
0.01359 \\
0.02870 \\
0.02018 \\
0.01249 \\
0.01324 \\
0.02051 \\
0.00499 \\
0.01035 \\
0.13671 \\
0.0152 \\
0.04468\end{array}$ & $\begin{array}{l}0.12453 \\
0.00461 \\
0.03947 \\
0.01613 \\
1.25167 \\
0.01941 \\
0.07903 \\
0.00266 \\
0.01255 \\
0.01734 \\
0.00600 \\
0.01519 \\
0.17165 \\
0.01343 \\
0.05137\end{array}$ & $\begin{array}{l}0.13204 \\
0.00645 \\
0.04433 \\
0.01833 \\
0.01477 \\
1.23385 \\
0.04400 \\
0.00470 \\
0.01594 \\
0.01748 \\
0.00613 \\
0.01686 \\
0.12849 \\
0.0145 \\
0.07130\end{array}$ & $\begin{array}{l}0.09022 \\
0.01952 \\
0.03726 \\
0.02675 \\
0.01856 \\
0.04445 \\
1.13942 \\
0.00931 \\
0.01609 \\
0.01837 \\
0.00499 \\
0.01330 \\
0.13462 \\
0.01213 \\
0.06332\end{array}$ & $\begin{array}{l}0.01093 \\
0.06820 \\
0.15190 \\
0.00542 \\
0.00909 \\
0.05576 \\
0.03205 \\
1.01256 \\
0.02407 \\
0.02660 \\
0.01374 \\
0.03770 \\
0.13256 \\
0.01208 \\
0.05706\end{array}$ & $\begin{array}{l}0.00839 \\
0.16110 \\
0.12370 \\
0.00912 \\
0.01064 \\
0.02220 \\
0.01997 \\
0.02200 \\
1.23847 \\
0.02362 \\
0.00928 \\
0.02355 \\
0.16836 \\
0.00921 \\
0.08920\end{array}$ & $\begin{array}{l}0.01360 \\
0.04273 \\
0.05063 \\
0.00693 \\
0.01373 \\
0.03089 \\
0.05732 \\
0.00939 \\
0.16402 \\
1.10073 \\
0.00385 \\
0.01235 \\
0.03746 \\
0.01078 \\
0.05775\end{array}$ \\
\hline & 11 & 12 & 13 & 14 & 15 & & & & & \\
\hline $\begin{array}{r}1 \\
2 \\
3 \\
4 \\
5 \\
6 \\
7 \\
8 \\
9 \\
10 \\
11 \\
12 \\
13 \\
14 \\
15\end{array}$ & $\begin{array}{l}0.01205 \\
0.04475 \\
0.04925 \\
0.00767 \\
0.06720 \\
0.02873 \\
0.11410 \\
0.11562\end{array}$ & $\begin{array}{l}0.00324 \\
0.00504 \\
0.15352 \\
0.00235 \\
0.01280 \\
0.01064 \\
0.00355 \\
0.01565\end{array}$ & $\begin{array}{l}0.00257 \\
0.00164 \\
0.01714 \\
0.00205 \\
0.00279 \\
0.01183 \\
0.00573 \\
0.00101 \\
0.00515 \\
0.00640 \\
0.00288 \\
0.00580 \\
1.01650 \\
0.05631 \\
0.03844\end{array}$ & $\begin{array}{l}0.00687 \\
0.00716 \\
0.19033 \\
0.00631 \\
0.00386 \\
0.01392 \\
0.04720 \\
0.00230 \\
0.00990 \\
0.01747 \\
0.01079 \\
0.00585 \\
0.09495 \\
1.00908 \\
0.04743\end{array}$ & $\begin{array}{l}0.00589 \\
0.00311 \\
0.01102 \\
0.00581 \\
0.00484 \\
0.01890 \\
0.01608 \\
0.00281 \\
0.01189 \\
0.02074 \\
0.01396 \\
0.00712 \\
0.05655 \\
0.00821 \\
1.12739\end{array}$ & & & & & \\
\hline
\end{tabular}


Cuadro A 3

MATRIZ $B$

\begin{tabular}{ll}
\hline Sectores & \\
\hline 1 & 0.74520 \\
2 & 0.53490 \\
3 & 0.53090 \\
4 & 0.36060 \\
5 & 0.41940 \\
6 & 0.41890 \\
7 & 0.36960 \\
8 & 0.46760 \\
9 & 0.38080 \\
10 & 0.40730 \\
11 & 0.43800 \\
12 & 0.68120 \\
13 & 0.87560 \\
14 & 0.62140 \\
15 & 0.75280 \\
\hline
\end{tabular}


Cuadro A 4

PRIMERA COMPONENTE PRINCíPAL

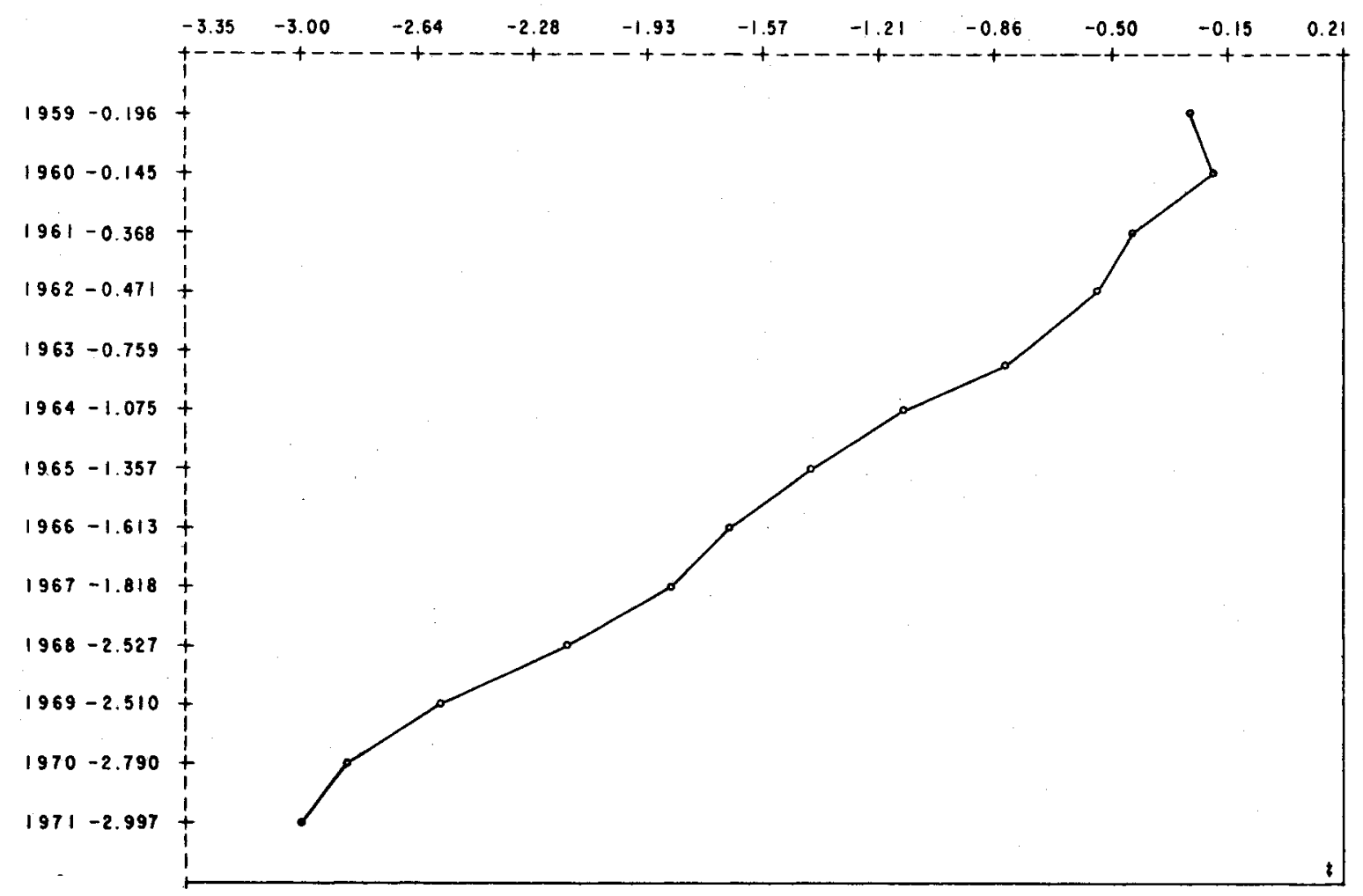


Cuadro A 5

SEGUNDA COMPONENTE PRINCIPAL

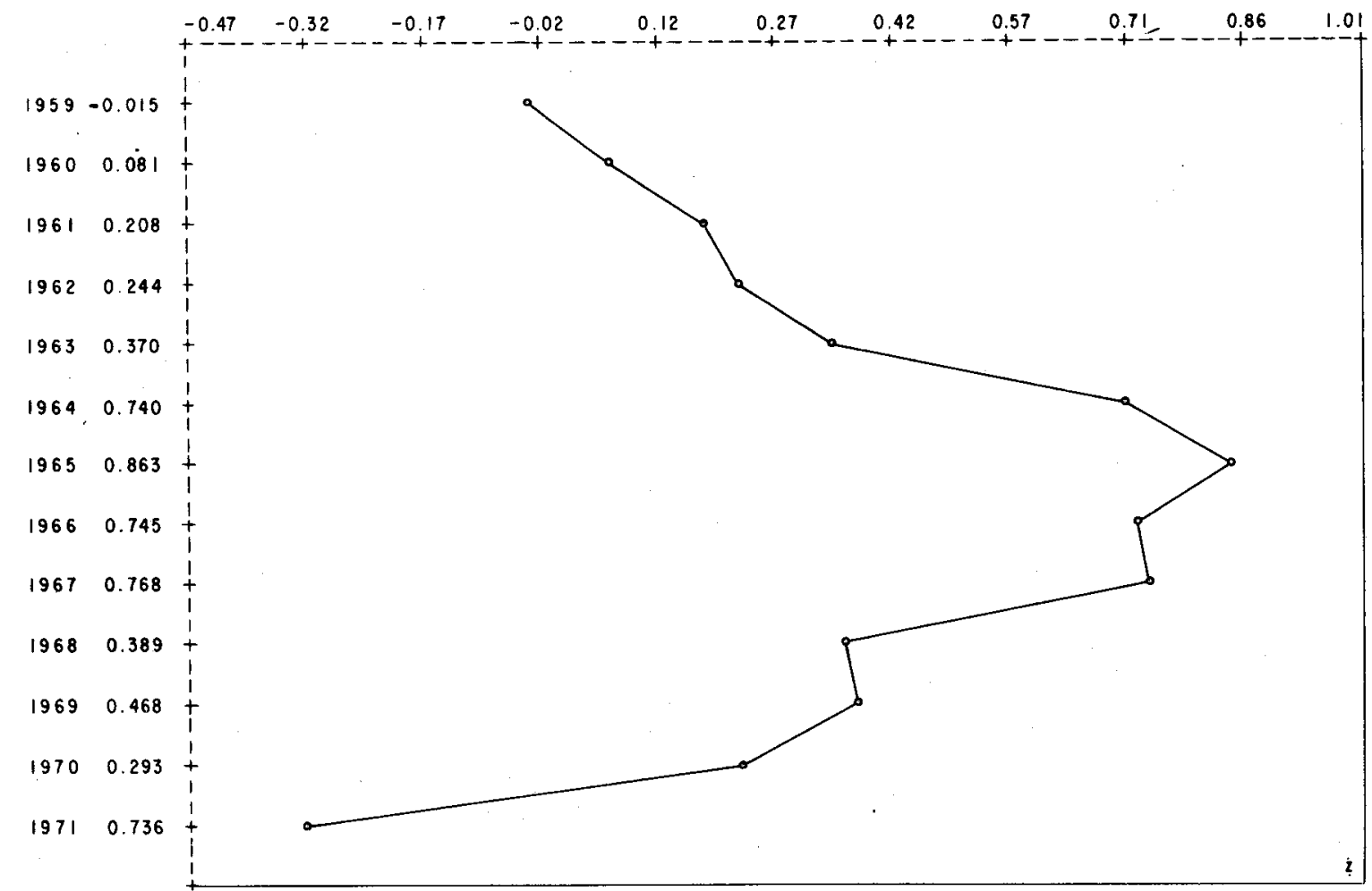


Cuadro A 6

TERCERA COMPONENTE PRINCIPAL

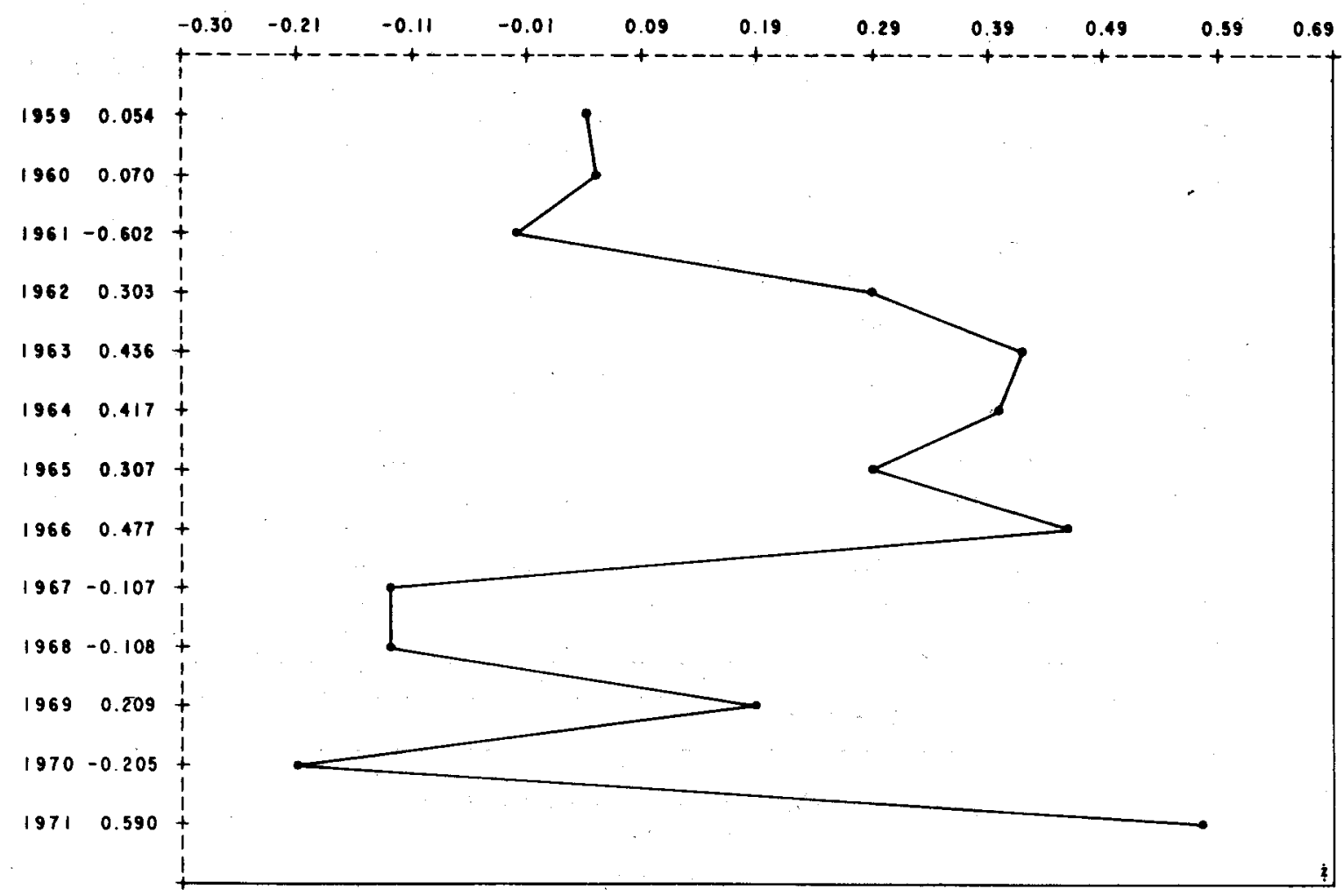


Cuadro A 7

Matriz de CONYERSIÓN DEL PRODUCTO, 1960

\begin{tabular}{|c|c|c|c|c|c|c|}
\hline \multirow{2}{*}{ Sectores } & \multicolumn{6}{|c|}{ Domande final } \\
\hline & 1 & 2 & 3. & 4 & 5 & 6 \\
\hline 1 & 0.21703 & 0.00939 & 0.07071 & 0.35354 & 0.05066 & 0.31646 \\
\hline 2 & 0.00538 & 0.00474 & 0.00513 & 0.23024 & 0.03057 & 0.03876 \\
\hline 3 & 0.08020 & 0.03514 & 0.02872 & 0.07075 & 0.03751 & 0.11005 \\
\hline 4 & 0.21912 & 0,00816 & 0.05660 & 0.26909 & 0.00948 & 0.12445 \\
\hline 5 & 0.09495 & 0.00543 & 0.11948 & 0.06477 & 0.00827 & 0.15672 \\
\hline 6 & 0.03133 & 0.02284 & 0.03851 & 0.02583 & 0.04297 & 0.07258 \\
\hline 7 & 0.05765 & 0.03568 & 0.02478 & 0.04590 & 0.02654 & 0.02328 \\
\hline 8 & 0.00504 & 0.01676 & 0.00316 & 0.01148 & 0.06105 & 0.00167 \\
\hline 9 & 0.01352 & 0.01198 & 0.01751 & 0.02284 & 0.09033 & 0.10126 \\
\hline 10 & 0.04487 & 0.04124 & 0.06615 & 0.02401 & 0.15297 & 0.15785 \\
\hline 11 & 0.00531 & 0.00540 & 0.01005 & 0.00532 & 0.51793 & 0.03299 \\
\hline 12 & 0.01254 & 0.02490 & 0.00800 & 0.01162 & $0.01409^{\circ}$ & 0.01033 \\
\hline 13 & 0.38090 & 0.04566 & 0.22936 & 0.16721 & 0.24501 & 0.13491 \\
\hline 14 & 0.06077 & 0.02531 & 0.06975 & 0.01119 & 0.01945 & 0.01020 \\
\hline 15 & 0.23311 & 0.05033 & 0.64902 & 0.05475 & 0.05925 & 0.04112 \\
\hline
\end{tabular}

" 1 = Consumo privado

$2=$ Cons

$3=$ Consumo de los turistas

$4=$ Exportaciones

$5=$ Inversión total fijada

$6=$ Cambios en inventarios 
Cuadro A 8

Matriz $H$

Ditribución INDUSTRIAL DE LA DEMANDA FINAL, 1960

\begin{tabular}{ccccccc}
\hline Sootore. & \multicolumn{7}{c}{ Denande 1 inal } \\
\cline { 2 - 7 } & 1 & 2 & 3 & 4 & 5 & 6 \\
\hline 1 & 0.12860 & 0.00158 & 0.03496 & 0.24609 & 0.03778 & 0.23416 \\
2 & 0.00002 & 0.00012 & 0.00000 & 0.17155 & 0.00096 & 0.01276 \\
3 & 0.01848 & 0.01407 & 0.00000 & 0.02188 & 0.00008 & 0.04721 \\
4 & 0.16983 & 0.00509 & 0.03975 & 0.20549 & 0.00160 & 0.08379 \\
5 & 0.06914 & 0.00279 & 0.09100 & 0.04308 & 0.00108 & 0.11570 \\
6 & 0.01106 & 0.01419 & 0.01676 & 0.00812 & 0.00200 & 0.04232 \\
7 & 0.03149 & 0.02632 & 0.00000 & 0.01889 & 0.00292 & 0.08060 \\
8 & 0.00086 & 0.01419 & 0.00000 & 0.00615 & 0.00104 & 0.02148 \\
9 & 0.00000 & 0.00291 & 0.00000 & 0.00786 & 0.00889 & 0.05572 \\
10 & 0.02843 & 0.03409 & 0.04502 & 0.00957 & 0.10131 & 0.13101 \\
11 & 0.00000 & 0.00340 & 0.00000 & 0.00000 & 0.51226 & 0.02786 \\
12 & 0.00468 & 0.02171 & 0.00000 & 0.00000 & 0.00461 & 0.00000 \\
13 & 0.31041 & 0.02693 & 0.16092 & 0.09608 & 0.14522 & 0.04551 \\
14 & 0.03733 & 0.02183 & 0.05316 & 0.00000 & 0.00349 & 0.00000 \\
15 & 0.17826 & 0.03603 & 0.55843 & 0.01855 & 0.01510 & 0.00000 \\
\hline
\end{tabular}

$$
\begin{aligned}
& 1=\text { Consumo privado } \\
& 2=\text { Consumo público } \\
& 3=\text { Consumo de los turistas } \\
& 4=\text { Exportaciones } \\
& 5=\text { Inversión total fijada } \\
& 6=\text { Cambio en inventarios }
\end{aligned}
$$

